\title{
Stenosing Tenosynovitis Due to Excessive Texting in an Adolescent Girl: A Case Report
}

\author{
Jason D. Johnson ${ }^{1} \quad$ Michael P. Gaspar ${ }^{2}$ Eon K. Shin ${ }^{2}$ \\ ${ }^{1}$ Department of Plastic and Reconstructive Surgery, Philadelphia \\ College of Osteopathic Medicine, Philadelphia, Pennsylvania, \\ United States \\ 2 Department of Orthopaedic Surgery, The Philadelphia Hand Center, \\ P.C., Thomas Jefferson University, Philadelphia, Pennsylvania, \\ United States \\ J Hand Microsurg 2016;8:45-48.
}

\begin{abstract}
Address for correspondence Michael P. Gaspar, MD, The Philadelphia Hand Center, P.C, 834 Chestnut Street, Suite G114, Philadelphia, PA 19107, United States (e-mail: michaelpgaspar@gmail.com).
\end{abstract}

\begin{abstract} Keywords

- stenosing tenosynovitis

- trigger thumb

- adolescent

- texting

- teenager

We present a case report of stenosing tenosynovitis of the flexor pollicis longus tendon in an adolescent girl who required surgical release after failing conservative measures. The patient had no other risk factors, aside from her excessive texting, which we postulate led to her condition. Although there have been a few reports of tendinitis and tenosynovitis secondary to texting, we believe this is the first in the literature to report trigger thumb requiring surgical release in an adolescent.
\end{abstract}

\section{Introduction}

Stenosing tenosynovitis (or "triggering") of the digits is most commonly found in the adult population, while occurrence in the pediatric age group is predominantly seen in the thumb. ${ }^{1,2}$ However, this condition is seen far less commonly in adolescents, with reports mostly limited to cases with a range of inciting causes. ${ }^{3-6}$ We present the case of an adolescent girl who developed stenosing tenosynovitis of her thumb from excessive texting. She was successfully treated with surgery after failing conservative measures.

\section{Case Report}

A 16-year-old right-hand-dominant girl was referred to our office by her primary care physician with a chief complaint of pain and limited motion of her right thumb. The patient stated that the pain initially began 1 year prior to her initial presentation, but had gradually worsened over the past 2 to 3 months. Over the same 1-year period, she reported a painful "snapping" sensation of the metacarpophalangeal (MP) joint with attempted thumb flexion which also progressed into a limited ability to flex MP joint in the recent months. At rest her pain was described as "mild-to-moderate," but worsened to "moderate-to-severe" with attempted MP motion. Her medical history was unremarkable, and she denied any history of trauma. She was a full-time high school student, worked part time as a waitress, and did not participate in organized athletic activities. She did not note any significant changes in type or amount of activities at school or requirements at work over the past few years. She had not undergone any form of treatment or activity modification prior to being seen at our clinic.

On physical examination, the patient was found to have tenderness to palpation over the A- 1 pulley and active triggering of the right thumb. There was also significant crepitus with active MP flexion, which was limited by roughly 20 degrees. Radiographs did not reveal any pathologic findings. Following a detailed discussion on conservative care options, in conjunction with the severity of her symptoms, the patient and her mother expressed a desire to proceed with a corticosteroid injection. She was administered $1.5 \mathrm{~mL}$ of a 50:50 corticosteroid mixture consisting of lidocaine hydrochloride 1\%(Hospira, Lake Forest, Illinois, United States) and betamethasone sodium phosphate and acetate $6 \mathrm{mg} / \mathrm{mL}$ (American Regent, Shirley, New York, United States) into the received

September 1, 2015

accepted after revision

November 20, 2015
C 2016 Society of Indian Hand \& Microsurgeons
DOI http://dx.doi.org/ 10.1055/s-0035-1571263. ISSN 0974-3227. 
flexor tendon sheath of the right thumb, which she tolerated well. Given the relatively unusual presentation of triggering in the thumb of an adolescent, the patient was asked to follow-up in 1 month for reevaluation.

When the patient returned 4 weeks later, her examination was significantly improved. In particular, she demonstrated full range of motion in her right thumb and noted that her pain had completely resolved. Though she still exhibited mild, persistent triggering, this was also significantly improved from her prior visit, and did not cause her any pain. In light of her clinical improvement, continued observation was recommended. However, when the patient returned to our office 1 month later, she complained of severe triggering, locking sensations, and difficulty with thumb MP extension. On examination, she again demonstrated significant tenderness to palpation over the A-1 pulley and active triggering. A second corticosteroid injection was administered at that time.

Six weeks later, the patient again returned with recurrent symptoms, and also noted that while the first injection provided relief of her symptoms for greater than 1 month, her most recent injection only did so for roughly 2 weeks. Upon further questioning, the patient conceded that she had texted extensively on her cellular phone over 1 year prior to her initial visit, and that she predominantly used her thumbs for texting ( - Table 1 ). Despite her attempts to reduce her texting volume, she was unable to adequately decrease her messaging, and her symptoms persisted. After extensive discussion with the patient and her mother, the decision was made for operative intervention. The patient was taken to the operating room 10 days later for a standard release of the

Table 1 Patient's cellular phone records showing her number of text messages sent per month

\begin{tabular}{|l|l|l|}
\hline Year & Month & $\begin{array}{l}\text { Text messages } \\
\text { sent }\end{array}$ \\
\hline 2011 & December & 9,967 \\
\hline 2012 & January & Not available \\
\cline { 2 - 3 } & February & 7,181 \\
\cline { 2 - 3 } & March & 10,193 \\
\cline { 2 - 3 } & April & 10,307 \\
\cline { 2 - 3 } & May & Not available \\
\cline { 2 - 3 } & June & 7,406 \\
\cline { 2 - 3 } & July & 7,085 \\
\cline { 2 - 3 } & August & 13,487 \\
\cline { 2 - 3 } & September & 13,001 \\
\cline { 2 - 3 } & October & 8,910 \\
\cline { 2 - 3 } & November & 4,325 \\
\cline { 2 - 3 } & December & 3,172 \\
\hline & Total number of texts (2012) & 85,067 \\
\hline & Average texts per month (2012) & 8,507 \\
\hline
\end{tabular}

${ }^{\mathrm{a}}$ Month of initial clinic visit.

bExcludes months for which the data were not available.
A-1 pulley to the right thumb flexor tendon sheath. Intraoperatively, she was found to have significant inflammation of the tissues surrounding the flexor pollicis longus (FPL) tendon (-Fig. 1). Following a tenosynovectomy procedure, the patient was awakened and was able to fully flex and extend the thumb without residual triggering.

At 2- and 6-week postoperative follow-up visits, the patient exhibited full range of motion of the thumb and a complete absence of residual triggering. She demonstrated mild tenderness to palpation over the flexor tendon sheath of the contralateral thumb. However, no overt triggering was noted, and continued observation was recommended.

\section{Discussion}

Stenosing tenosynovitis, commonly known as trigger finger or trigger thumb, is caused by a disparity in size between the flexor tendon sheath and its contents, resulting in abnormal tendon gliding. As the tendon attempts to pass through a stenotic sheath, it catches, thus producing pain and/or inability to flex or extend the digit effortlessly. ${ }^{7}$ Stenosing tenosynovitis is typically characterized by inflammatory changes in the retinacular sheath and peritendinous tissue rather than in the tenosynovium itself. ${ }^{8}$

Pediatric and adult trigger thumbs represent different entities requiring different treatment approaches. Despite some controversy, pediatric trigger thumb is considered an acquired condition caused by unique anatomic abnormalities. $^{1,7}$ Among the estimated $0.05 \%$ of all children diagnosed with trigger digits, $90 \%$ occur in the thumb, most commonly presenting at 2 years of age. ${ }^{7}$ Previous studies report a spontaneous resolution rate ranging from zero to $66 \%{ }^{1}$

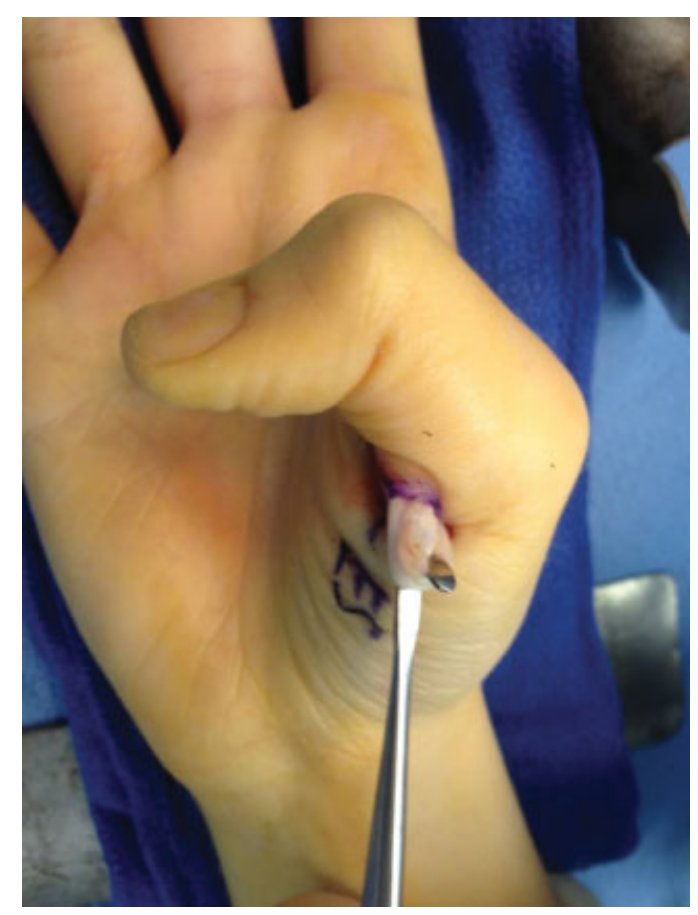

Fig. 1 Flexor tenosynovitis seen about the flexor pollicis longus (FPL) during trigger thumb surgery. 
In contrast, adult trigger thumb most commonly occurs in middle-aged women and may occur in conjunction with multiple trigger digits. Histological analysis has demonstrated fibrocartilaginous metaplasia, and it has been proposed this is secondary to repetitive friction between the tendon and sheath. ${ }^{8}$

In the adult population, treatment options typically include activity modification, anti-inflammatory medication, splint immobilization, corticosteroid injection, and surgical intervention. However, these same conservative treatments may have limited usefulness in the younger populations, as data regarding their efficacy have been inconclusive or lacking entirely in the pediatric and adolescent populations, respectively. ${ }^{1}$

Corticosteroid injection is a common first-line, minimally invasive treatment. Injection of the involved flexor tendon sheath provides long-term relief of symptoms in 60 to $92 \%$ of affected digits with up to three injections. ${ }^{7,8}$ A diminished response to injection has been associated consistently with an increased duration of symptoms, usually more than 4 to 6 months, and with an increasing number of injections. ${ }^{7,8}$ Rozental et al prospectively studied corticosteroid injections in 124 trigger digits in 199 patients with 35\% involving the thumb in an attempt to identify prognostic indicators of symptom recurrence. ${ }^{8}$ The results of open A-1 pulley release generally are excellent. Turowski et al, in a group of 59 patients treated by several surgeons, reported $97 \%$ complete resolution of triggering with no complications. ${ }^{9}$

According to a 2010 Kaiser Family Foundation study, 85\% of 15 - to 18 -year-olds own a personal cell phone, an increase of nearly $30 \%$ from just 5 years earlier. ${ }^{10}$ In a typical day, $46 \%$ of 8 - to 18 -year-olds reported sending text messages, with those who text estimated to send an average of 118 messages in a typical day. ${ }^{10}$ Fifteen- through 18 -year-olds spent the largest amount of time engaged in sending and receiving texts at 1 hour 51 minutes daily. ${ }^{10}$

Multiple reports have demonstrated the potential effects that texting may have on the development of tendonitis or tenosynovitis about the thumb, though most have focused mainly on pathology of the dorsal and/or radial side of the thumb. One case of "texting tendinitis" was reported in a 14-year-old girl who presented with tenderness overlying the extensor pollicis brevis, extensor pollicis longus (EPL), and abductor pollicis longus (APL) tendons of her dominant hand. ${ }^{11}$ That patient was successfully treated with a 2-week conservative treatment course of immobilization, anti-inflammatory medication, icing, and textingactivity modification. ${ }^{11}$ Similarly, Ashurst et al reported the case of a 48-year-old woman who developed bilateral De Quervain tenosynovitis from excessive texting treated successfully with a similar 3-week course of conservative treatment and limited texting. ${ }^{12}$ Along those lines, Menz reported on a 13-yearold girl with tenosynovitis of the APL/EPL at the level of the midforearm that resulted from text messaging. ${ }^{13}$ Her symptoms resolved after only a 2-day course of rest and topical antiinflammatory medication. ${ }^{13}$

In a larger study of 27 patients, Sharan and Ajeesh demonstrated that the largest proportions of symptoms seen were tendonitis or "myofascial pain" of the thenar musculature, but there were no reports of triggering of any digits. ${ }^{14}$ In an even larger study, Eapen and colleagues examined nearly 100 students who reported painful symptoms related to mobile phone use. ${ }^{15}$ They found that $40 \%$ of patients had positive findings on clinical exam, and $21 \%$ had ultrasound evidence of tendinopathy. Once again, however, all clinical exam findings were consistent with De Quervain-type pathology, and only $2 \%$ of patients were found to have fluid about the FPL on ultrasound without associated clinical symptoms. ${ }^{15}$

Using an electrogoniometer to measure thumb movements during mobile phone text messaging, Gustafsson et al found that subjects with musculoskeletal symptoms tended to have higher thumb movement velocities and fewer pauses in thumb movements compared with those without symptoms. ${ }^{16}$ In addition, they found that females had higher muscle activity in the extensor digitorum and APL when entering messages and tended to have greater thumb abduction, higher thumb movement velocities, and fewer pauses when compared with their male counterparts. ${ }^{16}$ of note, values for the FPL were not measured.

In our report, we present the unique case of a 16-year-old female patient with no risk factors for the development of stenosing tenosynovitis other than her substantial texting. Though the aforementioned studies report occurrences of tendonitis or tenosynovitis due to text messaging, nearly all reports are related to dorsoradial pathology, such as in De Quervain tenosynovitis. In addition, studies using electrogonimetry and ultrasound also focused on this region of pathology, suggesting that tenosynovitis involving the flexor aspect of the thumb is rarely appreciated. ${ }^{15,16}$ To our knowledge, this is the first report of stenosing tenosynovitis in a teenager that resulted in clinically disabling triggering of the thumb and ultimately required surgery. As the use of mobile devices increases while technology continues to progress, this rare clinical phenomenon may be of increasing importance in the near future. Thus, it is warranted to examine precipitating factors in our presented case in an effort to shed light on potential risk factors or preventative measures that may be implemented.

Based on the previously mentioned values from the Kaiser Family Foundation study, a typical teenager who uses text messaging services would average roughly 3,500 texts per month. ${ }^{10}$ According to - Table 1, our patient sent 7,085 texts per month at her lowest point and 13,487 at her peak prior to her initial visit in November 2012. Therefore, she was texting an average of 2 to 3.7 times more than the average texting teenager when her symptoms developed. In addition, she used a cell phone with depressible buttons instead of a touch screen (-Fig. 2), which likely increased the amount of muscle activity needed to enter a text message.

The previously mentioned studies in adolescent patients all demonstrated that conservative therapy with activity modification could successfully treat tenosynovitis symptoms. However, our patient's lack of adequate symptom relief with conservative treatment measures ultimately necessitated surgical intervention. She had no other risk factors that would predict development of stenosing tenosynovitis. Thus, it is reasonable to conclude that excessive texting alone may be a risk factor for 

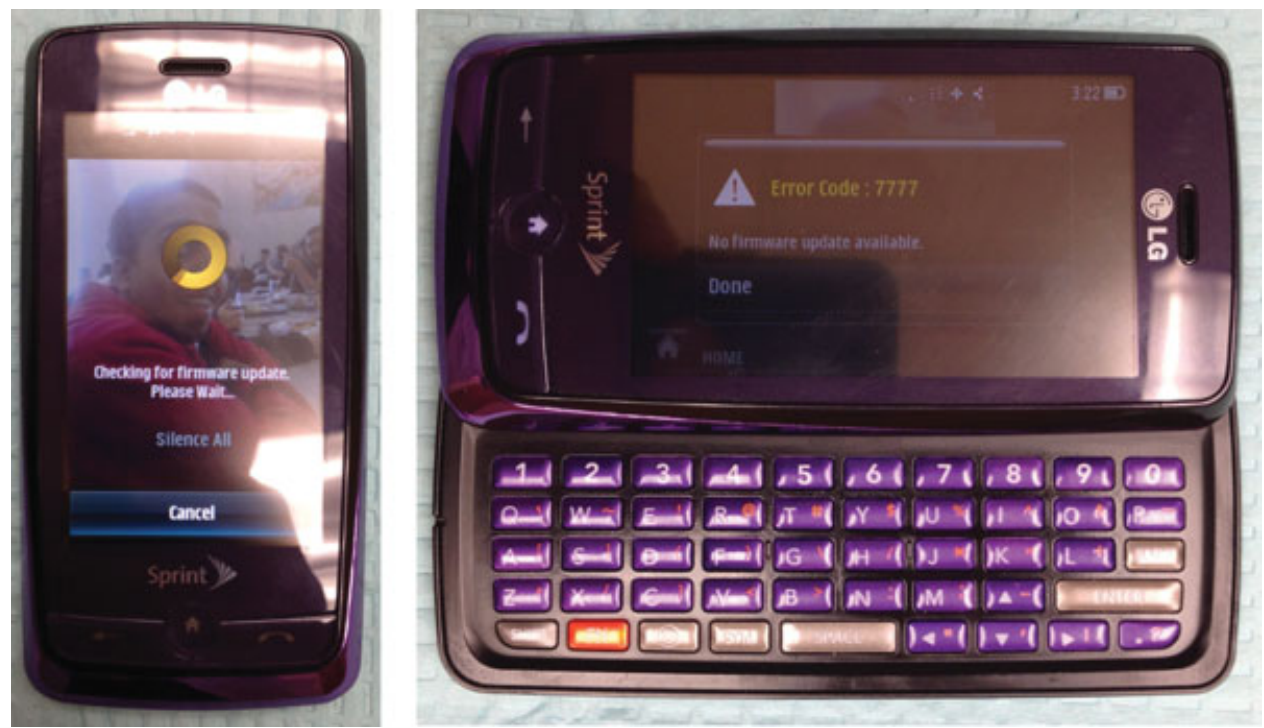

Fig. 2 The patient used an LG Rumor Touch cellular phone for text messaging. This particular model features a full QWERTY chiclet-style slide-out keyboard.

the development of stenosing tenosynovitis among adolescents. As cell phone use and texting continue to become more ubiquitous, the adolescent trigger thumb may become a more common finding, and patients and surgeons alike should be made aware of this possibility.

\section{Conflict of Interest \\ None.}

\section{Informed Consent}

The authors have obtained the patient's and her mother's informed written consent for print and electronic publication of the case report.

\section{References}

1 Shah AS, Bae DS. Management of pediatric trigger thumb and trigger finger. J Am Acad Orthop Surg 2012;20(4):206-213

2 Weilby A. Trigger finger. Incidence in children and adults and the possibility of a predisposition in certain age groups. Acta Orthop Scand 1970;41(4):419-427

3 Degreef I, Sciot R, De Smet L. Delayed post-traumatic trigger finger in a 14-year-old boy after blunt trauma. A case report. Acta Chir Belg 2007;107(6):731-732

4 Seiler JG III, Kerwin GA. Adolescent trigger finger secondary to post-traumatic chronic calcific tendinitis. J Hand Surg Am 1995; 20(3):425-427
5 Pampliega T, Arenas AJ. An unusual trigger finger. Acta Orthop Belg 1997;63(2):132-133

6 Espinoza DP, Heierli P, Raffoul W. Melorheostosis: a rare aetiology for trigger-finger. J Hand Surg Eur Vol 2010;35(4):318

7 Ryzewicz M, Wolf JM. Trigger digits: principles, management, and complications. J Hand Surg Am 2006;31(1):135-146

8 Rozental TD, Zurakowski D, Blazar PE. Trigger finger: prognostic indicators of recurrence following corticosteroid injection. J Bone Joint Surg Am 2008;90(8):1665-1672

9 Turowski GA, Zdankiewicz PD, Thomson JG. The results of surgical treatment of trigger finger. J Hand Surg Am 1997; 22(1):145-149

10 Rideout VJ, Foehr UG, Roberts DF. Generation M2: media in the lives of 8- to 18-year-olds. A Kaiser Family Foundation study. Available at: http://www.kff.org/entmedia/upload/8010.pdf. Published January 2010. Accessed June 28, 2013

11 Williams IW, Kennedy BS. Texting tendinitis in a teenager. J Fam Pract 2011;60(2):66-67, 1, 67

12 Ashurst JV, Turco DA, Lieb BE. Tenosynovitis caused by texting: an emerging disease. J Am Osteopath Assoc 2010;110(5): 294-296

13 Menz RJ. “Texting” tendinitis. Med J Aust 2005;182(6):308

14 Sharan D, Ajeesh PS. Risk factors and clinical features of text message injuries. Work 2012;41(Suppl 1):1145-1148

15 Eapen C, Kumar B, Bhat AK, Venugopal A. Extensor pollicis longus injury in addition to De Quervain's with text messaging on mobile phones. J Clin Diagn Res 2014;8(11): LC01-LC04

16 Gustafsson E, Johnson PW, Hagberg M. Thumb postures and physical loads during mobile phone use - a comparison of young adults with and without musculoskeletal symptoms. J Electromyogr Kinesiol 2010;20(1):127-135 